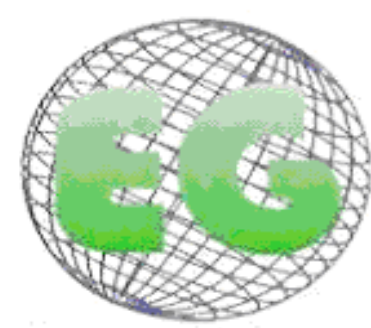

ISSN 1695-6141

$\mathrm{N}^{\circ} 24$
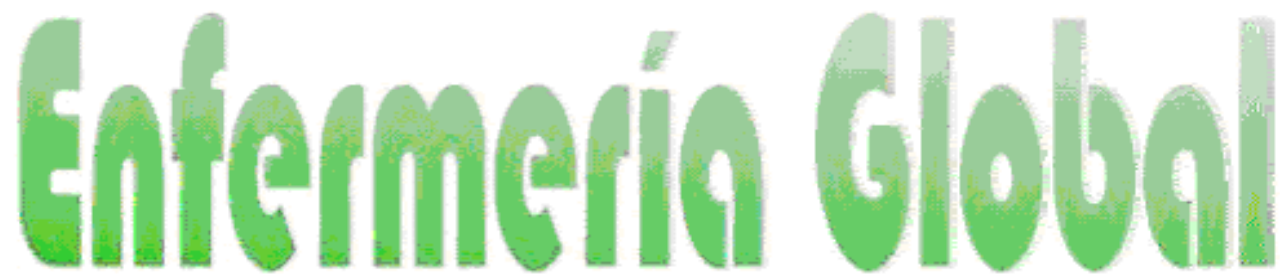

Revista electrónica trimestral de Enfermería

Octubre 2011

www.um.es/egloball

\title{
La Planificación Estratégica como proceso de integración de un equipo de salud
}

Strategic planning and integration of a health team

\author{
*Leal de Valor, DY. **Bolívar de Muñoz, ME. **Castillo Torrealba, CO. \\ "Profesora Asistente. ** Profesora /r Asociado. Escuela de Enfermería " Dra. Gladys Román de Cisneros" ,
}

Facultad de Ciencias de la Salud. Universidad de Carabobo. Venezuela.

Palabras clave: Planificación Estratégica, Integración, Atención en Salud

Keywords: Strategic Planning, Integration, Health Care

\section{RESUMEN}

La Planificación Estratégica es un proceso que conduce al capital humano a establecer metas organizacionales, definir estrategias y políticas para el logro de esas metas, desarrollar planes para asegurar la implantación de las estrategias y así obtener los fines buscados. Este estudio está enmarcado en la línea de investigación: Administración de los Recursos Humanos, cuyo propósito fue diseñar un plan estratégico de integración del equipo de salud para optimizar la atención a los usuarios que acuden a un ambulatorio del IVSS“ en el Estado Carabobo. Este estudio se caracterizó como un proyecto factible, basado en una investigación de campo de tipo descriptivo, la muestra fue de tipo intencional y estuvo conformada por 17 personas que corresponden a estrategas del equipo de salud del ambulatorio. Para la recolección de la información se utilizó la técnica de encuesta y como instrumento el cuestionario estructurado en 30 ítems, el cual fue validado por juicio de expertos, quienes aportaron resultados positivos; para determinar la confiabilidad interna se calculó el coeficiente Alpha de Cronbach, obteniendo un valor significativo de $0,83 \%$. Los datos fueron analizados mediante la técnica de análisis porcentual simple y fueron presentados por cuadros y gráficas circulares. Se propone un plan de integración fundamentado en la planificación estratégica con el objetivo de proporcionar las herramientas necesarias que le permitan superar las debilidades y amenazas y aprovechar las fortalezas y oportunidades para optimizar la atención en salud a los usuarios que acuden al ambulatorio.

\section{ABSTRACT}

Strategic Planning is a process that leads to human capital to establish organizational goals, strategies and policies for achieving those goals, develop plans to ensure implementation of strategies and obtain the ends sought. This study is framed in the research: Human Resource Management, whose purpose was to design a strategic plan for integration of the healthcare team to optimize service to users who visited the outpatient IVSS" in the state of Carabobo. This study was characterized as a feasible project, based on field research and descriptive, the sample was intentional and consisted of 17 people who belong to health strategists of the ambulance team. For data collection technique was used as an instrument survey and structured questionnaire of 30 items, which was validated by experts, who provided positive results to determine the internal reliability was calculated Cronbach alpha coefficient, obtaining significant value of $0.83 \%$. The data were analyzed using simple percentage analysis technique and were presented by tables and circle graphs. It proposes an 
integration plan based on strategic planning in order to provide the tools necessary to enable it to overcome the weaknesses and threats and capitalize on the strengths and opportunities to improving health care for the users who come to the ambulance.

\section{INTRODUCCIÓN}

En esta época de complejidades, cambios e incertidumbres como la vivida hoy día, se percibe a la Administración como una de las áreas mas importantes de la actividad humana, dirigida hacia la producción de bienes y servicios, en las cuales un Gerente Administrativo debe aplicar una serie de funciones en forma sistemática como son: Planificar, organizar, dirigir y controlar, lo cual le llevará a la solución de situaciones previamente diagnosticadas y jerarquizadas en una organización.

La Planificación Estratégica se conceptualiza como un sistema gerencial mediante el cual quienes toman las decisiones en una organización obtienen, procesan y analizan la información pertinente interna y externa, con el fin de evaluar la situación presente de una empresa, así como su nivel de competitividad, con el propósito de anticipar y decidir sobre el direccionamiento de la institución hacia el futuro ${ }^{(1)}$.

En Venezuela, la creciente complejidad de los sistemas de salud y de las organizaciones que participan en ellos demandan el desarrollo de nuevas visiones, conocimientos y habilidades en el desempeño administrativo del capital humano ${ }^{(2)}$, desde una perspectiva global y estratégica para establecer metas y objetivos ${ }^{(3)}$ y por consiguiente en el proceso de la toma de decisiones de las organizaciones de salud a la que pertenecen. El sistema gerencial en los diferentes nivelas de atención de salud se han abocado a tratar de solucionar la grave crisis institucional en los servicios de salud ${ }^{(4)}$, como lo es el Ministerio de Salud y Desarrollo Social (MSDS), que representa la primera y mayor institución pública dedicada a la salud del país, cuenta con el $75 \%$ personal de todas las instituciones relacionadas con la salud, con una estructura organizacional de cuatro niveles jerárquicos, del cual emanan todas las decisiones en los centros de salud, produciéndose asincronías y lentitud en todos los procesos, lo cual repercute en la planificación y ejecución de acciones y programas destinados a mejorar la atención a los usuarios que asisten a los diferentes centros de salud, obteniendo como resultado incumplimiento de los mismos ${ }^{(5)}$

Otra institución pública prestadora de salud es el Instituto Venezolano de los Seguros Sociales (IVSS), tiene una cobertura del $35 \%$ en 17 entidades del país, a través de Centros Ambulatorios, Hospitales y Servicios Odontológicos, con personalidad jurídica propia y cuya misión es brindar protección de la seguridad social a todos los beneficiarios en las contingencias de maternidad, vejez, sobre vivencia, enfermedad, accidentes, incapacidad, invalidez, nupcias, muerte, retiro y cesantía o perdida de empleo, de manera oportuna y con calidad de excelencia en el servicio prestado, dentro del marco legal que lo regula y evidentemente con un modo de atención diferente ${ }^{(6)}$.

Generalmente, las instituciones reflejan un patrón de comportamiento similar, ya que poseen una concentración de recursos en los grandes Hospitales con una planificación inadecuada a las necesidades reales de salud de la población asistida, déficit de personal de salud (por necesidad de servicio y/o reposo médico), déficit de medicamentos, huelgas de trabajadores, luchas gremiales, falta de motivación y de integración del equipo de salud al cumplimiento de metas y objetivos, planes y programas, que se puede calificar como un colapso, ya que la amenaza de la paralización general de los servicios es constante. 
El estado de Carabobo constituye el primer estado industrial del país, tiene un mayor incremento anual de su población y por ende de la clase trabajadora ${ }^{(7)}$, después del Distrito Capital, es el estado más poblado, situación que contribuye a agravar la crisis de salud; hay ausencia de planificación de política regional en salud integral; no obstante, existen las emanadas del MSDS, se evidencian desajustes administrativos, inexistencia de liderazgo, falta de motivación al personal, gerentes que no poseen las competencias ni la formación gerencial, inherencia bipartidista en la selección y designación de las autoridades gerenciales.

En lo que a Planificación se refiere, se constató que el diseño de planes por parte de los integrantes del equipo de salud no se cumple con la debida planificación, existe desconocimiento de metas y objetivos organizacionales, improvisaciones en las actividades administrativas, exceso de trabajo y por consiguiente agotamiento laboral en el personal por la recarga de funciones y tareas. El objetivo de esta investigación permitió diseñar un Plan Estratégico de Integración del Equipo de Salud para optimizar del proceso de atención a los usuarios que asisten al ambulatorio.

Es importante mencionar el estudio realizado por Godoy ${ }^{(8)}$ quien presentó un plan administrativo basado en la planificación estratégica situacional en el sector educativo media, diversificada y profesional como alternativa de acción viable al diagnóstico que resultó de un estudio de campo descriptivo que determinó que la funcionabilidad del plan es regular, siendo las dimensiones: reuniones e informaciones, supervisión y solicitud de recursos humanos y materiales a entes externos, que ameritaron la planificación de acciones específicas contenidas en la propuesta.

Por otra parte, Solís ${ }^{(9)}$ se planteó analizar la planificación estratégica utilizada por el personal directivo como herramienta básica para optimizar el recurso humano en los planteles privados de Valencia. Los resultados evidencian que los directores y docentes de los planteles privados de la III Etapa de Educación Básica, en su mayoría conocen y participan en el proceso de planificación estratégica, y que existen instituciones que están organizando y formándose en planificación estratégica como instrumento en la administración de sus recursos.

Debe señalarse también, la investigación de Flores ${ }^{(10)}$ quien planteó un plan estratégico para el fortalecimiento de la relación escuela-comunidad, concluyó proponiendo un plan fundamentado en la planificación estratégica dirigido a los docentes con el propósito de proporcionar las herramientas necesarias para superar las dificultades y alcanzar una integración positiva con todos los miembros de la comunidad.

Igualmente, el estudio realizado por Arévalo(11), cuyo objetivo fue proponer un plan estratégico para el fortalecimiento de la gestión ambiental. Los resultados se confirmaron con la incorporación a escala local con una estrategia en la definición y ejecución de una política que asegure la calidad de vida para los ciudadanos, asegurando que las alternativas e impactos indirectos y acumulativos que se produzcan sean adecuadamente considerados.

La Planificación Estratégica constituye un proceso gerencial que desplaza el énfasis en el ¿qué lograr? (objetivos) al ¿qué hacer? (estrategias) y se concentra solo en aquellos objetivos factibles de lograr y en qué áreas competir, en correspondencia con las oportunidades y amenazas que ofrece el entorno. Este proceso consiste en responder: ¿A dónde queremos ir?, ¿Dónde estamos hoy?, ¿A dónde debemos ir?, ¿A dónde podemos ir?, ¿A dónde iremos?, ¿Cómo estamos llegando a nuestras metas?- Y está formada por seis (06) componentes fundamentales: los estrategas, el direccionamiento estratégico, el 
diagnóstico estratégico, las opciones estratégicas, formulación estratégica y auditoría estratégica $^{(12)}$

Los Estrategas son los funcionarios a quienes les corresponde la definición de los objetivos y políticas de las organizaciones, deben ser lo más participativo posible de manera que todos se sientan comprometidos con los valores, visión, misión y objetivos de la organización y deben ser excelentes comunicadores, con una gran capacidad analítica, con dotes de liderazgo, buenas relaciones interpersonales.

El Direccionamiento Estratégico está integrado por los Principios Corporativos (valores, creencias y normas que deben ser compartidos por todos sus miembros), la Visión (señala el rumbo, define la dirección de la organización y sirve de guía en la formación de estrategias) y la Misión (incluye la formulación de los propósitos de la organización).

El Diagnóstico Estratégico es el marco de referencia para el análisis actual de una organización, tanto internamente como frente a su entorno, es la respuesta de ¿dónde estábamos?, ¿dónde estamos hoy?

Las Opciones Estratégicas son las expectativas que la organización tiene para anticipar tanto sus oportunidades y amenazas, como sus fortalezas y debilidades, para ello partiendo de la matriz DOFA y del análisis de la vulnerabilidad efectuado por el diagnóstico.

La Formulación Estratégica constituyen los planes de acción concretos, con definición responsable, ya que es indispensable proyectar cada uno de los proyectos estratégicos, definir los objetivos y las estrategias de cada área funcional dentro de los mismos.

La Auditoría Estratégica indica que el desempeño de la organización debe ser monitoreado y auditado tomando en cuenta los objetivos, los planes de acción y el presupuesto estratégico; motivo por el cual se definen unos índices que permiten medir este desempeño que debe realizarse en forma periódica, de manera que retroalimente oportunamente el proceso de la planificación estratégica y puedan introducirse los ajustes o modificación de la situación requerida.

Marco de referencia para la Planificación Estratégica Sanitaria. En esta perspectiva, la planificación estratégica sanitaria debe ser a partir de las necesidades de salud, tanto si se trata de servicios, como los programas de prevención y asistencia, ya que la salud puede definirse como la capacidad del individuo para mantener un estado de necesidades sociales, en el que él está razonablemente estable, indemne de profundas incomodidades, insatisfacciones, enfermedad o incapacidad y de comportarse de tal forma que asegure la supervivencia de su especie tan bien como su propia realización personal ${ }^{(13)}$.

Es por ello que los indicadores negativos de la salud (mortalidad y morbilidad) es y será el principal objeto de preocupación para el planificador de salud; de allí que la función esencial de un planificador consiste en establecer planes y prever para el futuro a partir de la situación actual que representa la diferencia entre lo que existe y lo que es deseable en términos de salud, servicios y recursos.

Integración y Eficacia del Equipo de Salud. Uno de los mecanismos más efectivos para elevar la eficiencia organizacional es la integración del trabajo en equipo, el cual estimula al individuo en su sentido de pertenencia a la empresa, ya que un Equipo Eficaz es aquel que logra alcanzar sus metas con ideas innovadoras, se adapta al cambio cuando es necesario y 
sus miembros están altamente comprometidos, es valiosamente estimado por los gerentes que reconocen y recompensan su desempeño.

Bajo esta concepción el Equipo de Salud debe desempeñarse como promotor a la integración y participación, debe saber cuáles son sus necesidades para poder entender la de sus clientes, usuarios y pacientes, todo ello en vía de integrar y optimizar la atención en salud.

\section{MATERIALES Y MÈTODOS}

Este estudio se enmarcó en la modalidad de proyecto factible ${ }^{(14)}$, se estructuró en tres (03) fases: fase I: diagnóstico; fase II: estudio de factibilidad, factibilidad operativa/ técnica, factibilidad financiera, y culminó con la fase III: diseño de la propuesta

\section{Fase I}

Diagnóstico: Esta fase consistió en determinar la necesidad diseñar un Plan Estratégico de Integración del Equipo de Salud para optimizar el proceso de atención a los usuarios que asisten al ambulatorio a través de una encuesta tipo cuestionario estructurada en dos (02) partes y dirigida a una muestra intencional que representó el 35\% de la población total que estuvo constituida por 48 personas que laboran en los diferentes servicios y departamentos de la institución distribuidos por estratos y denominación.

La primera parte relacionada con los datos socio laborales referidos al sexo, edad, nivel académico y años de experiencia en el sector salud. La segunda parte presentó 30 ítems, para cada una de las cuales hay cinco (05) opciones de respuesta en la escala de Likert con los criterios de: Siempre (S), Frecuentemente (FR), Algunas Veces (AV), Raras Veces (RV), Nunca(N). El instrumento contiene instrucciones específicas para orientar a los elementos muéstrales como ubicar las respuestas y elaborados en base a la operacionalizaciòn de la variable.

La validación del instrumento se plasmó mediante la revisión de expertos con titulo de Magister, quienes emitieron su opinión en cuanto a la tabla de variables desglosada en sus dimensiones e indicadores que permitieron perfeccionarlo. La confiabilidad interna se efectuó por medio de una prueba piloto aplicada a un grupo conformado por diez (10) integrantes del equipo de salud no pertenecientes a la muestra seleccionada, luego se calculó el coeficiente Alpha de Cronbach obteniéndose un valor significativo de $0,83 \%$.

El análisis estadístico de los datos se realizó mediante la frecuencia alcanzada por cada ítem, los porcentajes se representaron en cuadros y gráficos circulares. Los resultados obtenidos evidencian que existe un alto porcentaje de desconocimiento por parte del equipo de salud sobre las pautas de planificación que impiden el adecuado desempeño de sus funciones.

\section{Fase II.}

Estudio de Factibilidad: En esta fase se procedió a estudiar la factibilidad operativa, la factibilidad técnica y la factibilidad financiera para la aplicación de la propuesta al equipo de salud. 
Factibilidad Operativa/ Técnica: Se consideraron todos aquellos recursos materiales necesarios y recursos humanos que participaron, y se reflexionó sobre todas aquellas actividades que fueron necesarias para lograr los objetivos y que permitió evaluarla.

Factibilidad Financiera: Se contó con el apoyo institucional, quién consideró todos los recursos económicos y financieros necesarios que permitieron desarrollar o llevar a cabo las actividades para elaborar la propuesta.

\section{Fase III}

Diseño de la Propuesta: Se elaboró Propuesta titulada "Plan Estratégico de Integración del Equipo de salud, para optimizar la atención de los usuarios que asisten a un ambulatorio del IVSS". Se le describió los objetivos, los principios corporativos, se especificaron sus características: ser implementada bajo la modalidad de Talleres, integradora, flexible, es pertinente y presencial; su Plan de Acción, así como también estructurada en cinco (5) unidades y cada unidad con su respectivo programa. Todo ello, teniendo como base para su aplicación que la salud no es un concepto abstracto, sino un bien común de las personas, es un derecho moral y legal del que todos deben beneficiarse tal y como lo establece la Constitución de la República Bolivariana de Venezuela en su artículo 83, parte del 84, 85 y 86 (15); y para ello el equipo de de salud de las diferentes organizaciones debe ser el más óptimo.

\section{DISCUSIÓN DE LOS RESULTADOS}

El proceso de información se inició con las características sociales y laborales de la muestra seleccionada. La contextualización de estos resultados permitió arrojar las siguientes realidades:

Datos personales y profesionales: El $87 \%$ del personal corresponde al sexo femenino, el $82 \%$ del personal son mayores de 43 años, el 100\% del personal tiene de nivel académico estudios universitarios y el $82 \%$ tiene más de 23 años en el sector salud. Tal y como se refleja en el gráfico1.

Gráfico № 1 Resultado porcentual de los Datos Personales y Profesionales del Equipo de Salud

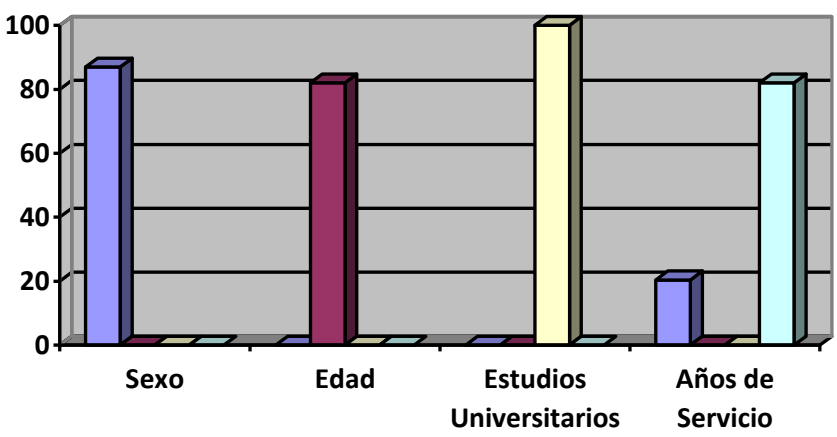

En relación a las opciones de respuesta en la escala de Likert con los criterios de: Siempre (S), Frecuentemente (FR), Algunas Veces (AV), Raras Veces (RV), Nunca(N), resultó que: el $18 \%$ afirma que siempre se establecen metas, el $70 \%$ alega que las pautas de planificación las establece la organización, el $48 \%$ asevera que nunca se identifica con la misión de la 
institución, el $70 \%$ refiere que nunca proponen estrategias que faciliten el cumplimiento de los objetivos establecidos. Información representada en el gráfico 2.

Gráfico № 2.- Resultado porcentual de las opciones de respuestas de las variables investigadas

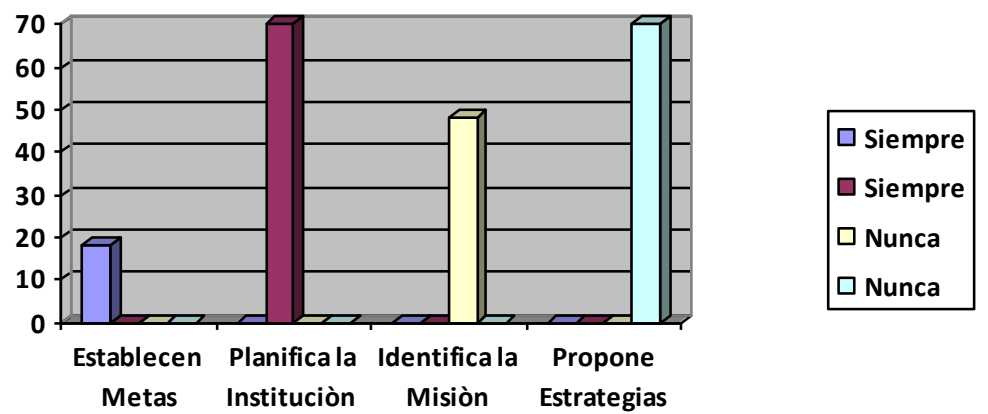

También se determinó que solo el $41 \%$ siempre tienen sentido de cooperación con sus compañeros de trabajo, el $76 \%$ afirma que siempre está dispuesto a asistir a talleres, cursos de formación y otros que les permitan ampliar sus conocimientos, el $41 \%$ indica que solo alguna veces realiza planificación de las actividades diarias, el 35\% asume que nunca participa en conjunto con otros integrantes en la toma de decisiones de las actividades del ambulatorio. Datos representados en el gráfico 3.

Gráfico № 3.- Resultado porcentual de las opciones de respuestas de las variables investigadas

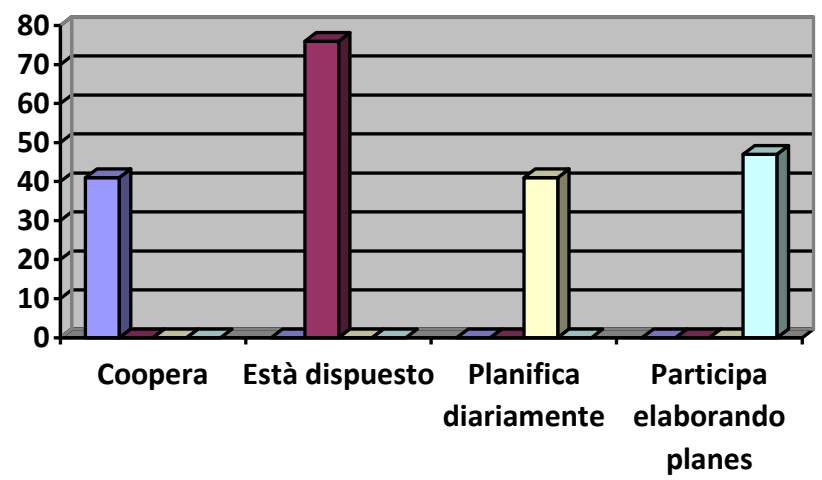

\begin{tabular}{|l|}
\hline$\square$ Siempre \\
$\square$ Siempre \\
$\square$ Algunas veces \\
$\square$ Algunas veces
\end{tabular}

Igualmente se comprobó que el $47 \%$ asume participar algunas veces en la elaboración de planes en su servicio, consulta y/o departamento, el $40 \%$ afirma que algunas veces participa en la redefinición de los planes en función de mejorar los resultados, el $47 \%$ dice que solo algunas veces propone estrategias que faciliten la integración del equipo de salud y finalmente el $52 \%$ afirma que siempre estaría dispuesto a trabajar en equipo. Información mostrada en el gráfico 4. 
Gráfico №4. Resultado porcentual de las opciones de respuestas de las variables investigadas

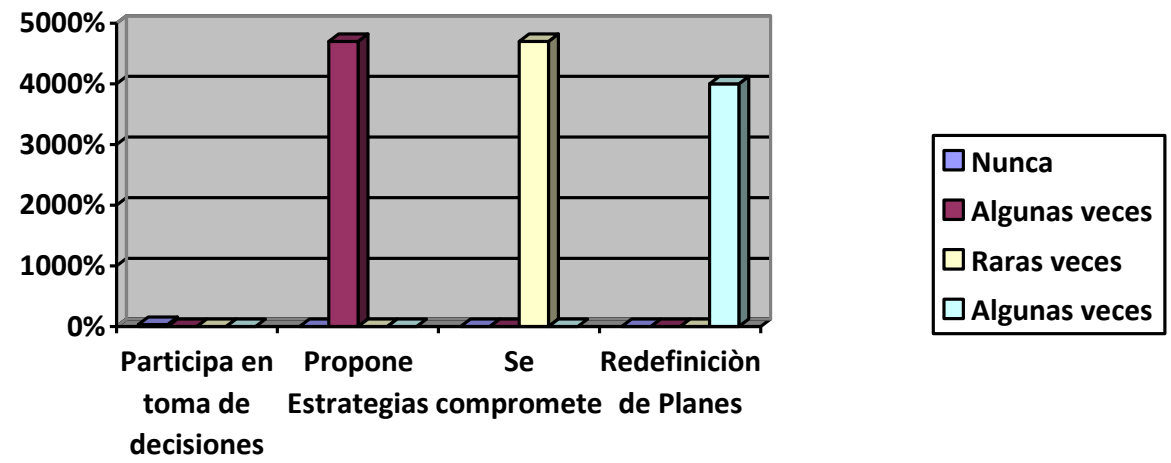

\section{CONCLUSIONES}

Una vez finalizada la investigación, se llegó a las siguientes conclusiones:

Se reconoce que el equipo de salud es el capital humano más valioso que tiene el ambulatorio.

El equipo de salud debe concienciarse y asumir el rol, según los requerimientos y necesidades de los usuarios.

Es necesario que para el logro de un buen desempeño, se requiere seleccionar los mejores estrategas, y por ello se debe crear una cultura de calidad de atención en la organización.

En cuanto a la dimensión integración, el proceso de comunicación presenta debilidades en todos los niveles.

La participación activa en la gestión es baja, ya que el personal de salud no participa y por consiguiente está ausente el sentido de pertenencia en relación a los logros.

El equipo de salud no esta formado en relación a los procesos de planificación, esto influye en la poca participación de las actividades, así como también en su desempeño.

En la institución el talento humano está dispuesto a recibir preparación y formación acorde a las funciones que deben cumplir.

\section{REFERENCIAS BIBLIOGRÁFICAS}

1. Serna, H. Gerencia Estratégica. Planeación y Gestión -Teoría - Metodología. 7ma. edición. 3R Editores. Bogotá, Colombia. 2002.

2. Szilangyc, A. An empirical test of causal inference between rol perpeption, satisfaction with Word, performance and organizational level. Personnel psychology 30(3): 375-388. 1977. 
3. Certo, S. Administración Moderna. Octava Edición. Bogotá. D.C. 2001

4. Malavè, J. Gerencia en Salud, un modelo innovador. Ediciones IESA. Fundación Cisneros Benavides. Caracas. Venezuela. 1995.

5. Pérez, A y Jae, M. Descentralización del sector salud, transferencia de servicios y reestructuración del MSAS: Propuestas y escenarios. Informe 4. Papel de Trabajo Inédito. PNUD-Copre. Caracas. Venezuela. 1994.

6. Folleto interno del IVSS. Emanado de la Oficina Central del IVSS. Altagracia. Caracas. Venezuela. 2000.

7. Oficina Central de Información y Estadística (OCEI). Caracas. Venezuela.2000.

8. Godoy, S. La Planificación Estratégica Situacional en el Proceso Administrativo de Educación Media Diversificada y Profesional. Trabajo de Grado de Maestría. Universidad de Carabobo. Valencia. Venezuela.1996

9. Felipe, S. La Planificación Estratégica como herramienta en la administración del recurso humano en los planteles privados. Trabajo de Grado de Maestría. Universidad de Carabobo. Valencia. Venezuela. 1998.

10. Flores, B. Plan Estratégico para el fortalecimiento de la relación Escuela- Comunidad en Educación Básica. Trabajo de Grado de Maestría. Universidad Rafael Urdaneta. Maracaibo. Venezuela. 2003.

11. Arévalo, L. Plan Estratégico para el Fortalecimiento de la Gestión Ambiental Municipal del Estado Guárico. Trabajo de Grado de Maestría. Universidad Rómulo Gallegos. San Juan de los Morros. Venezuela. 2003.

12. Serna, H. Op. Cit.

13. Pineault, M. La planificación sanitaria: conceptos, métodos, estrategias. Ediciones Masson. S.A. Barcelona. España. 1990.

14. Universidad Pedagógica Experimental Libertador. Vicerrectorado de Investigación y Postgrado. Manual de Trabajos de Grado de Maestrías y Tesis Doctorales. Caracas. Venezuela.1998.

15. Constitución de la República Bolivariana de Venezuela. Gaceta Oficial № Diciembre. Caracas. Venezuela.199 